\title{
Family Physicians' Knowledge of Commonly Overused Treatments and Tests
}

\author{
Douglas Maurer, DO, MPH, Mark Stephens, MD, Brian Reamy, MD, \\ Brian Crownover, MD, Paul Crawford, MD, and Tammy Chang, MD
}

Purpose: In 2010, several primary care physician organizations drafted the "Promoting Good Stewardship in Clinical Practice" list for family medicine to advance the appropriate use of 5 commonly ordered treatments and tests. The goal was to achieve excellent patient care while appropriately conserving health care resources. The purpose of this study was to assess knowledge regarding the appropriate use of these treatments and tests among the memberships of the Uniformed Services Academy of Family Physicians (USAFP) and the Council of Academic Family Medicine (CAFM).

Methods: An online, cross-sectional survey of all family physician members of the USAFP and the CAFM was conducted. The survey consisted of 5 clinical cases administered as part of a larger omnibus CAFM Educational Research Alliance survey and a separate USAFP membership survey. The primary outcome was correct answers to clinical scenarios.

Results: The response rate was $11 \%(n=236$ of 2165$)$ for members of USAFP and $47 \%(n=519$ of 1099) for CAFM members, with an overall response rate of $23 \%(n=755$ of 3264$)$. Overall, respondents answered $78 \%$ of cases correctly (USAFP 79\%, CAFM 78\%; no significant difference). In rank order, respondents were most likely to answer the Papanicolaou test question correctly $(90.1 \%)$, followed by the low-back pain $(86.7 \%)$, the dual-energy $X$-ray absorptiometry screening $(85.4 \%)$, the sinusitis (66.5\%), and the cardiac screening questions (61.4\%).

Conclusion: Among the family physicians surveyed, knowledge about the appropriate use of commonly overused tests and treatments was high. Two clinical scenarios (sinusitis and cardiac screening) demonstrate areas where further education could improve care and reduce costs. (J Am Board Fam Med 2014;27:699-703.)

Keywords: Cardiovascular Diseases, Diagnostic Techniques and Procedures, Low-Back Pain, Prevention and Control, Primary Health Care, Sinusitis

Health care spending in the United States continues to increase and now accounts for nearly $20 \%$ of the nation's gross domestic product. ${ }^{1}$ Most experts

This article was externally peer reviewed.

Submitted 6 January 2014; revised 26 April 2014; accepted 2 May 2014.

From the Madigan Army Medical Center Family Medicine Residency, Tacoma, WA (DM); the Uniformed Services University of the Health Sciences, Bethesda, MD (MS, $\mathrm{BR}$ ); the Nellis Air Force Base Family Medicine Residency, Nellis AFB, NV (BC, PC); and the Department of Family

Medicine, University of Michigan, Ann Arbor (TC).

Funding: none.

Conflict of interest: none declared.

Prior Presentations: This work has been presented as a poster at the North American Primary Care Research Group Practice-based Research Network Conference, Bethesda, MD (June 18-19, 2013).

Corresponding author: Douglas M. Maurer, DO, MPH, Department of Family Medicine, Madigan Army Medical Center, 7040 Jackson Ave, Tacoma, WA 98433 (E-mail: douglasmaurer@aol.com). consider this level of health care spending unsustainable. Health economists identify unnecessary diagnostic and screening tests as a primary driver of this spending. ${ }^{2}$ Many patients request or receive "routine" (but unnecessary) medical tests. The National Physicians Alliance and several other primary care physician organizations have initiated programs to promote the appropriate use of screening and diagnostic tests to facilitate high-quality, costconscious care. ${ }^{3}$

The Military Health System is a large health care organization. Serving almost 10 million beneficiaries worldwide, ${ }^{4}$ the Military Health System benefits from a strong core of primary care physicians available to every enrollee. The Uniformed Services Academy of Family Physicians (USAFP) is the medical society that serves as the professional home for the largest group of military physicians. 
Comprising uniformed family physicians and civilian family physicians working in military facilities around the globe, the USAFP has more than 1600 active members.

The Council of Academic Family Medicine (CAFM) services more than 1000 family physicians who are engaged primarily in academic practice. The CAFM Educational Research Alliance (CERA) is a research organization comprising multiple partners within academic family medicine. CERA partners include the Society of Teachers of Family Medicine, the Association of Departments of Family Medicine, and the Association of Family Medicine Residency Directors. This study was designed to investigate specifically baseline knowledge regarding appropriate utilization of 5 common diagnostic and screening tests among USAFP and CAFM members.

\section{Methods}

Following institutional review board approval from the Uniformed Services University of the Health Sciences, we developed 5 standard cases that are commonly associated with unnecessary testing based on common primary care scenarios (see http://www. aafp.org/about/initiatives/choosing-wisely.html). We used a focused cadre of educational leaders in family medicine, a medical school family medicine department chair, a medical school dean, 2 current family medicine program directors, and a family medicine associate program director. We used standard evidence-based guidelines to design the case scenarios. ${ }^{5-10}$ All questions were closed ended (see Appendix 1). Items were included to gain basic descriptive information, including sex, age, service, and practice setting.

Using a modification of the approach described by Dillman, ${ }^{11}$ we E-mailed invitations to potential participants. E-mail lists were obtained from both USAFP and CERA. The introductory E-mail message explained the rationale of the study and advised interested participants to click on an embedded hyperlink to access the secure, anonymous, 5-question survey. A second request was sent to nonrespondents 1 week later. A third and final request was emailed to nonrespondents 1 month after the initial request.

We used general demographic statistics to analyze sample characteristics (age range, sex, military experience, years since medical school/internship/
Table 1. Demographics of the Uniformed Services Academy of Family Physicians (USAFP) and Council of Academic Family Medicine (CAFM) Respondents

\begin{tabular}{lcc}
\hline & USAFP $(\mathrm{n}=2165)$ & CAFM $(\mathrm{n}=1099)$ \\
\hline Respondents & $236(11 \%)$ & $519(47 \%)$ \\
Male sex & $161(71 \%)$ & $301(58 \%)$ \\
Age $<40$ years & $109(46 \%)$ & $83(16 \%)$ \\
Active duty & $172(75 \%)$ & $\mathrm{NA}$ \\
Academic practice & $52(22 \%)$ & $265(51 \%)$ \\
$\quad$ setting & & \\
\hline
\end{tabular}

NA, not applicable.

residency, duty title, practice setting, practice organization, hospital size, full-time equivalent status, types of learners taught). $\chi^{2}$ Analysis was used to examine correlational relationships between answers and demographic variables.

\section{Results}

Of 2165 eligible members of USAFP, 236 responded, resulting in a response rate of $11 \%$. Of 1099 eligible members of CAFM, 519 responded, resulting in a response rate of $47 \%$. This resulted in a total eligible population of 3264 , with 755 respondents, for an overall response rate of $23 \%$. There were significantly more male respondents in the USAFP compared with CAFM ( $71 \%$ vs $58 \%$; $P<.001)$. This is consistent with military physician populations, which tend to have more men than women. In addition, USAFP members were significantly younger compared with CAFM members ( $46 \%$ vs $16 \%$ aged $<40$ years; $P<.0001$ ). Of the USAFP respondents, 172 (75\%) were still on active duty. A nonteaching military clinic was the most common practice setting for USAFP members (77\%), followed by an academic practice setting (22\%). Of the CAFM respondents, an academic practice setting was the most common practice setting $(51 \%)$, whereas only $1 \%$ reported they were in a nonacademic practice setting. Table 1 described the demographics of study participants. There were some incomplete surveys. From the USAFP data, there was one incomplete survey. From the CAFM data, there were 16 incomplete surveys. These data were included in the final analysis because in all cases the fifth case was the question skipped. All other cases and demographic data were completed by all respondents.

While CAFM respondents answered more questions correctly than USAFP respondents, the dif- 
ference was not statistically significant. On question 1 (sinusitis), $66.3 \%$ of CAFM respondents answered correctly versus $66.9 \%$ of USAFP respondents $(P=.934)$. On question 2 (preoperative evaluation), $59.6 \%$ of CAFM respondents answered correctly versus $65.3 \%$ of USAFP respondents $(P=.147)$. On question 3 (Papanicolaou smear screening), $90.8 \%$ of CAFM respondents answered correctly versus $88.6 \%$ of USAFP respondents $(P=.360)$. On question 4 (dual-energy $\mathrm{X}$-ray absorptiometry screening), $86.6 \%$ of CAFM respondents answered correctly versus $82.6 \%$ of USAFP respondents $(P=.182)$. On question 5 (low-back pain), $87.6 \%$ of CAFM respondents answered correctly versus $84.7 \%$ of USAFP respondents $(P=.296)$.

When examining the combined data, $66.5 \%$ of respondents correctly answered the sinusitis question; $61.4 \%$ correctly answered the preoperative evaluation question; $90.1 \%$ correctly answered the Papanicolaou smear screening question; 85.4\% correctly answered the dual-energy X-ray absorptiometry screening question; and $86.7 \%$ correctly answered the low-back pain question.

\section{Discussion}

Our study examined the baseline knowledge of USAFP and CAFM members regarding the appropriate use of common diagnostic and screening tests, recently highlighted as overused by the Choosing Wisely campaign. ${ }^{3,12} \mathrm{We}$ did not find a significant difference in the baseline knowledge between the 2 groups. Overall, physicians were correct in $60 \%$ to $90 \%$ of their individual responses; CAFM members slightly trended toward more correct responses than USAFP members.

Response trends were also similar between the 2 groups. Both groups answered incorrectly more often on questions dealing with the management of sinusitis and preoperative clearance. Considering the frequency with which rhinosinusitis is encountered in primary care ( $4 \%$ of all outpatient visits), our results suggest the need for ongoing education in the conservative management of uncomplicated sinus disease. ${ }^{13}$ Similarly, many individuals seek preoperative clearance from their primary care physicians before routine surgeries and receive avoidable electrocardiograms. Our results indicate that resisting the urge to order unnecessary items is difficult and prevalent; the consequences involve potential medical harm and unnecessary escalation of costs. ${ }^{14,15}$ For example, if an unnecessary preoperative electrocardiogram shows nonspecific changes, the likelihood is high that the patient would be referred for additional unnecessary cardiac testing. If antibiotics are needlessly prescribed for routine sinus disease, potential harms include adverse drug reactions or unintended increases in antibiotic resistance. ${ }^{16}$ Guiding appropriate decision making in the context of these 2 clinical scenarios represents an area where ongoing physician education may be helpful.

Our study showed encouraging results for bone density screening, cervical cancer screening, and low-back pain. This is particularly heartening given that cytology screening guidelines have changed significantly in the past 5 years. Given the reported overuse of diagnostic imaging in the context of low-back pain, ${ }^{17}$ the fact that $>90 \%$ of respondents correctly identified the appropriate use of imaging is quite hopeful as well.

Study limitations include a low response rate from the USAFP membership. However, a low response rate is common in physician surveys. Another limitation is that all the case scenarios used for the study were purely hypothetical. It is possible that respondents would answer differently in "real-world" clinical situations. All scenarios were, however, based on the Choosing Wisely campaign recommendations to intentionally approximate conditions commonly seen in primary care. ${ }^{18}$

\section{Conclusion}

In general, baseline knowledge of appropriate utilization of diagnostic and screening tests was high among the sampled USAFP and CAFM populations. We found no significant difference in the overall knowledge between the 2 groups using 5 common clinical scenarios modeled after the Choosing Wisely campaign recommendations. Two case scenarios (sinusitis and routine preoperative electrocardiographic screening) were areas where further education seems to be indicated.

The opinions or assertions contained herein are the private views of the authors and are not to be construed as official or reflecting the views of the Department of the Army or the Department of Defense.

\section{References}

1. The World Bank. Data. Health expenditures, total (\% of GDP)-2010. Available from: http://data.worldbank. 
org/indicator/SH.XPD.TOTL.ZS. Accessed July 19, 2014.

2. Smith S, Newhouse JP, Freeland MS. Income. Insurance, and technology: why does health spending outpace economic growth? Health Aff (Millwood) 2009;28:1276-84.

3. Good Stewardship Working Group. The "top 5" lists in primary care: meeting the responsibility of professionalism. Arch Intern Med 2011;171:1385-90.

4. Military Health System Stakeholders Report. 2012. Available from: http://www.health.mil/ /media/ MHS/Report\%20Files/Optimized\%202012_MHS_ Stakeholders_Report120207.ashx. Accessed July 24, 2014.

5. U.S. Preventive Services Task Force. Screening for osteoporosis: U.S. preventive services task force recommendation statement. Ann Intern Med 2011;154:356-64.

6. Rosenfeld RM, Andes D, Bhattacharyya N, et al. Clinical practice guideline: adult sinusitis. Otolaryngol Head Neck Surg 2007;137(3 Suppl):S1-31.

7. Huntzinger A. Guidelines for the diagnosis and management of rhinosinusitis in adults. Am Fam Physician 2007;76:1718-24.

8. Moyer VA; U.S. Preventive Services Task Force. Screening for coronary heart disease with electrocardiography: U.S. Preventive Services Task Force recommendation statement. Ann Intern Med 2012;157: 512-8.

9. Chou R, Fu R, Carrino JA, Deyo RA. Imaging strategies for low-back pain: systematic review and metaanalysis. Lancet 2009;373:463-72.

10. Guide to clinical preventive services, 2012. AHRQ Publication No. 12-05154, October 2012. Rockville, MD: Agency for Healthcare Research and Quality; 2012. Available from: http://www.ahrq.gov/professionals/ clinicians-providers/guidelines-recommendations/guide/ index.html. Accessed July 24, 2014.

11. Dillman DA. Mail and telephone surveys: the total design method. New York: John Wiley \& Sons; 1978.

12. Choosing Wisely campaign. Lists. Philadelphia: American Board of Internal Medicine; 2014. Available from: http://www.choosingwisely.org/doctorpatient-lists/. Accessed July 19, 2014.

13. Cherry DK, Woodwell DA, Rechtsteiner EA. National Ambulatory Medical Care Survey: 2005 summary. Adv Data 2007;(387):1-39.

14. Moynihan R, Doust J, Henry D. Preventing overdiagnosis: how to stop harming the healthy. BMJ 2012; 344:e3502.

15. Paterniti DA, Fancher TL, Cipri CS, Timmermans S, Heritage J, Kravitz RL. Getting to "no": strategies primary care physicians use to deny patient requests. Arch Intern Med 2010;170:381-8.

16. Chow AW, Benninger MS, Brook I, et al.; Infectious Diseases Society of America. IDSA clinical practice guideline for acute bacterial rhinosinusitis in children and adults. Clin Infect Dis 2012;54:e72-112.
17. Srinivas SV, Deyo RA, Berger ZD. Application of "less is more" to low back pain. Arch Intern Med 2012;172:1016-20.

18. American College of Emergency Physicians. Five things physicians and patients should question. Philadelphia: American Board of Internal Medicine (ABIM) Foundation. Available from: http:// www.choosingwisely.org/doctor-patient-lists/ american-college-of-emergency-physicians/. Accessed July 19, 2014.

\section{Appendix 1}

The Five Standard Cases Used in the Study

1. A 55-year-old female presents for sinus pain, similar to prior episodes; she reports a frequent history of bacterial sinusitis. She typically receives amoxicillin or azithromycin, which give rapid relief. According to your current practice patterns, which of the following presentations is MOST likely to trigger you to prescribe antibiotics?

A. Fever to $101^{\circ} \mathrm{F}$, ear pain, postnasal drip, and severe cough $\times 5$ days

B. Unilateral purulent nasal discharge with moderate sinus fullness and pain $\times 5$ days

C. Persistent sinus congestion/fullness, postnasal drip, and cough at day 7

D. Headache, nasal discharge, and sinus pain that initially improved but is now worsening at day 7

2. A 55-year-old male presents for his annual physical. He is generally healthy. He takes a daily multivitamin and saw palmetto for "prostate support." He is taking no other medications and reports no problems. He is active physically and has had no symptoms (chest pain, shortness of breath, or exercise intolerance). You have been working with him for years and know him well. During examination, you find an inguinal hernia on the right side. He says that it has caused him no symptoms but that he "probably should get it fixed." His BP is $120 / 76 \mathrm{mmHg}$, pulse is $66 \mathrm{bpm}$, and body mass index is 24 ; the remainder of his physical examination is normal. His lipoprotein profile was done last year: total cholesterol $=175$; high-density lipoprotein $=66$; high-density lipoprotein $=88$. He is quite attuned with wanting to be in "tip-top" shape and asks for your recommendations to get him ready for surgery. As part of this visit, would you recommend an electrocardiogram as part of your current routine practice for preoperative clearance?

A. Yes 
B. No

3. In your normal practice, which of the following patients would receive a Papanicolaou test? (Assume none have received a Papanicolaou test within the past 3 years.)

A. A 66-year-old sexually active, monogamous woman with no history of cervical dysplasia

B. A 46-year-old sexually active, monogamous woman who has had a total hysterectomy

C. A 46-year-old sexually active, monogamous woman with no history of cervical dysplasia

D. An 18-year-old student who requests birth control pills before leaving for college

4. In your normal practice you would MOST LIKELY perform a DEXA scan to screen for osteoporosis on which one of the following patients?

A. A 54-year-old obese, postmenopausal woman with no family history of osteoporosis

B. A 54-year-old obese, postmenopausal woman with no family history of osteoporosis who has been taking 10 mg methylprednisolone daily for 1 year
C. A 54-year-old obese, postmenopausal woman who smokes 1 pack of cigarettes daily and has no family history of osteoporosis

D. 54-year-old obese, postmenopausal Asian woman with no family history of osteoporosis

5. In your current practice, you would MOST LIKELY order plain films of the lumbar spine on which of the following patients with lowback pain?

A. A 25-year-old female with low-back pain for 3 days after lifting furniture. She has no "red flag" symptoms.

B. A 25-year-old female with low-back pain for 4 weeks after lifting furniture. She has no "red flag" symptoms.

C. A 25-year-old female with low-back pain for 2 weeks after lifting furniture. She has no "red flag" symptoms.

D. A 25-year-old female with low-back pain for 8 weeks after lifting furniture. She has no "red flag" symptoms. 